A Motivational Framework for Psychopathy: Towards a Reconceptualization of the Disorder

Groat, L. L.

Shane, M. S.

The University of Ontario Institute of Technology, Oshawa, ON, Canada

Corresponding author:

Groat, L. L.

2000 Simcoe St. N

University of Ontario Institute of Technology

Forensic Psychology - CIBC Building Downtown - DTC619

Oshawa, ON, L1H 7K4

Email: Lindsay.Groat@uoit.ca

\title{
Notes:
}

This manuscript was accepted for publication on November 7, 2019 and is currently awaiting publications.

This version of the article may not completely replicate the final authoritative version published in European Psychologist. It is not the version of record and is therefore not suitable for citation. Please do not copy or cite without the permission of the author(s).

Both authors contributed equally to this publication, and authorship was chosen by mutual agreement. 


\begin{abstract}
The link between psychopathy and violence has been well documented. Estimates have psychopathic offenders responsible for as much as $40 \%$ of violence-related crime (e.g., Hare, 1999; extrapolated from Serin, 1991), and show rates of violent recidivism up to five times higher than non-psychopathic offenders (Hemphill, Hare, \& Wong, 1998). Existing theories of the disorder argue that this violence stems from core insensitivity to emotional/aversive information, or from core inability to optimally allocate processing resources in complex environments. However, some newer findings have been difficult for existing theories to assimilate; moreover, successful treatment programs based off current conceptualizations have been slow to develop With this in mind, the current paper proposes a new motivational framework for considering psychopathy, within which the disorder is conceptualized as stemming from more strategic, motivated processes. The paper begins by reviewing traditional theories of psychopathy and highlighting their explanatory strengths and limitations. The proposed motivational framework is then outlined, and a supportive rationale for the framework provided. Next, the paper undertakes a selective review of some of the most empiricallysupported features of the disorder, in order to highlight how these features may be productively reformulated within a motivational framework. Finally, the paper suggests several methods through which an empirical evaluation of the proposed ideas may be undertaken, and explores potential implications of a motivational framework for next-generation rehabilitation and treatment opportunities.
\end{abstract}

Keywords: psychopathy, violence, theory, motivation, emotion regulation, treatment 


\section{A Motivational Framework for Psychopathy: Towards a Reconceptualization of the}

\section{Disorder}

Psychopathy is a disorder characterized by interpersonal/affective (e.g. grandiosity, shallow affect), and behavioural/criminogenic (e.g. impulsivity, criminal versatility)

characteristics (Cleckley, 1941; Hare, 1991) that combine to form an individual who engages in a disproportionate amount of societal violence. Estimates have psychopathic offenders responsible for as much as 40\% of violence-related crime (e.g., Hare, 1999; extrapolated from Serin, 1991), and show rates of violent recidivism up to five times higher than non-psychopathic offenders (Hemphill, Hare, \& Wong, 1998). By some accounts, the violence committed by psychopathic individuals is also more instrumental (e.g. Williamson, Hare, \& Wong, 1987; but see Blais, Solodukhin, \& Forth, 2014), and more callous (e.g. Williamson, Hare \& Wong, 1987) than their nonpsychopathic counterparts. Particularly troubling, the disorder has remained resistant to most contemporary treatment efforts, potentially because our understanding of the disorder's underpinnings remains incomplete. A more complete understanding may thus aid development of novel therapeutic regimens, and may lead, in turn, to a reduction in violence associated with the disorder.

Traditional theories of psychopathy have largely posited core deficits in fundamental emotional and/or cognitive processes, and can be grouped into two broad categories. 'Emotiondeficit theories' argue for core reductions in emotional experience/reactivity, whereas 'cognitivedeficit theories' argue for a core inability to adaptively allocate processing resources. The empirical work in support of abnormal emotional/cognitive processing has been fairly convincing, to be sure; however, work indicating that these abnormalities result from core incapacities to manifest normative responses is far less developed. Moreover, evidence from a 
variety of sources has accumulated to suggest that at least some psychopathic individuals can show normative emotional experiences under certain specifically-designed contexts (e.g. Newman, Curtin, Bertsch, \& Baskin-Sommers, 2010; Shane \& Groat, 2018). This evidence has been difficult to assimilate into existing theories, and updated, more nuanced theories have only recently begun accounting for these phenomena (e.g. Hamilton et al., 2015; Shane \& Peterson, 2004; Vitale, Kosson, Resch \& Newman, 2018). With this in mind, we propose here a novel motivational framework for conceptualizing psychopathy that shares several features with existing theories, but introduces the notion that the disorder may include strategic, motivated components. We begin by reviewing existing theories, highlighting several of their strengths and limitations, before detailing the proposed motivational framework. Next, we undertake a selective reconsideration of the existing literature, to demonstrate how this motivational framework may afford a productive reconceptualization of fundamental characteristics of the disorder. Our intentions are not to put forth a singular, formalized motivational theory of psychopathy, nor to undertake an exhaustive review of the extant literature. Rather, we seek only to encourage consideration of a motivational framework, and demonstrate how such consideration may promote a productive, alternate understanding of the disorder. Finally, we consider potential avenues for empirically testing the proposed ideas, and undertake a preliminary consideration of potential treatment opportunities that a motivational framework may encourage.

\section{Traditional Theories of Psychopathy}

One of the earliest descriptions of psychopathy derives from Cleckley (1941) who suggested that although psychopathic individuals are capable of outwardly mimicking complex emotions, they nonetheless suffer a general poverty of affective experience. Cleckley 
hypothesized that this inability to experience deep, complex emotions prevents full integration of the emotional consequences of the psychopath's actions, thereby hindering adaptive punishmentbased learning and socialization. Several subsequent theories have taken their cue from Cleckley, but have argued that this affective poverty may be specific to select (generally negatively-valent) emotional states. Perhaps most influential, the Low Fear Hypothesis posits innately low levels of fear (or what Lykken termed 'the fear quotient'), that prevent development of fear-punishment associations of magnitude sufficient to influence motivational systems (Lykken, 1957; see also Fowles, 1980, who elaborates on the purported physiology). . Alternately, Blair (1995; see also Marsh \& Cardinale, 2012) has argued that psychopathic individuals may be unable to recognize or adapt to distress- and/or fear-related cues displayed by others, which may predispose one towards violent transgressions. While each theory focuses on a different affective process, common to each emotion-deficit theory is the suggestion of a fundamental inability to fully experience or process negatively-valent or aversive stimuli.

In contrast, another family of theories - which we will refer to here as 'cognitive-deficit theories' - argue that the emotional deficits seen in psychopathy are not due to core affectspecific dysfunction, but rather to more subtle limitations of the psychopath's early attentional processing. Most influential has been the response modulation hypothesis, which argues that psychopathic individuals suffer an inability to optimally allocate processing resources, particularly within complex environments (e.g. Patterson \& Newman, 1993). Specifically, once psychopathic individuals are focused on a dominant response set, they become unable to process information incongruent with their current goal set. The response modulation hypothesis has spawned several additional models, which may be viewed as iterative evolutions on the original hypothesis. The attentional bottleneck hypothesis (Baskin-Sommers, Curtin \& Newman, 2011) 
introduced the notion of a theoretical limitation to the psychopathic individual's information

processing pipeline, which restricts them to processing complex, multi-component information in serial fashion. Subsequently, the Impaired Integration framework elaborated on the manner in which this seriality may interfere with the speeded integration of set-incongruent information (Hamilton, Hiatt-Racer, and Newman, 2015). Of importance, these attention-based theories suggest that psychopathic individuals should be capable of processing affective stimuli when attention is focused on emotion-related cues (e.g. Newman, et al., 2010). Nonetheless, these theories retain the broader notion of specific (cognitive) deficits beyond the ability of the individual to control.

\section{A Motivational Framework for Psychopathy}

Importantly, while considerable empirical work confirms that psychopathic individuals show aberrant emotional/cognitive processing, confirmation that these alterations are the result of core incapacities is strikingly absent (see Shane \& Groat, 2018). Thus, while the psychopath does not typically manifest normative responses, it may be inaccurate to say that they cannot do so. In addition, several key features of the disorder are difficult for existing 'deficit models' to explain. For instance, emotion-based theories have difficulty explaining several of the more cognitive-based features of the disorder, including the psychopathic individual's superior filtering of goal-irrelevant information (e.g. Zeier, Maxwell \& Newman, 2009; Hiatt, Schmitt \& Newman, 2004); cognitive-based theories, in contrast, require some calisthenics to explain some of the emotion-based findings, such as the psychopathic individual's reduced identification of certain emotional faces (e.g. Hastings, Tangney \& Stuewig, 2008); and both groups of theories have some difficulty explaining why the specific emotion(s) that appear deficient show considerable between-study variability (i.e. the low fear hypothesis doesn't predict reduced 
sadness; the violence inhibition model doesn't predict reduced startle to non-facial images; the response modulation hypothesis hasn't explained why the incongruency of task-irrelevant emotional information would show between-study variability). Though these difficulties do not negate the possibility that each theory holds important truths, they do suggest that no existing theory perfectly explains all features of the disorder. The slow development and implementation of successful treatment protocols based on these theories further underlines this point (though see Baskin-Sommers, Curtin \& Newman, 2015), and suggests that additional conceptualizations may be beneficial.

An alternate perspective, described here, may posit the psychopathic individuals' reduced processing of emotional information as stemming from differences in motivation, rather than capacity. For instance, whereas traditional theories of the disorder argue that the psychopathic individual is incapable of experiencing sufficient emotion, or is incapable of adaptively allocating processing resources, a motivational framework may posit a reduced inclination to fully process aversive stimuli. Such inclination could conceivably manifest as indifference - i.e. psychopathic individuals may have no motivation to allocate processing resources towards negatively-valent/aversive stimuli; or as active avoidance - i.e. psychopathic individuals may have negative motivation to allocate processing resources towards negatively-valent/aversive stimuli, and thus avoid such allocation in order to deflect the impact that full processing of the aversive stimuli would otherwise entail. Moreover, these motivational stances could conceivably occur at any point along the spectrum of automaticity: through underlying differences in innate motivational tendencies; through fully conscious, deliberate avoidance of stimuli deemed aversive (c.f. Gross, 1998b); or through a reinforced response tendency that has become increasingly habituated over time (see Vitale et al., 2018). Indeed, the level of automaticity may 
be expected to show considerable variability across both individual and context. Regardless, a primary foundation of this motivational framework is that the psychopathic individual may be viewed less as helpless victim of their physiological constitution, and more as one who actively even if habitually - engages in the processing of, and interaction with, environmental stimuli.

This motivational framework draws heavily from work detailing the goal-relevant nature of emotion and emotional regulation, including seminal work by Maya Tamir and colleagues who's instrumental approach of emotion regulation posits emotion as experienced in the service of one's self-defined goals (e.g. Tamir, Chiu, \& Gross, 2007; Tamir, Ford \& Gilliam, 2013). Central to this instrumental approach is the notion that people are motivated to feel certain emotions, based not only on the hedonic value of the emotion, but also with regard to which emotions they would like to experience, and how well that emotion fits with their current goal set (e.g. Tamir, 2016). Thus, when an individual has an expectation that a given emotion will be useful, preference to experience that emotion - even negative emotions - may be expected to increase. The strength of this instrumental approach is at least two-fold: it lays the groundwork for explaining and predicting when one may be motivated (or unmotivated) to experience a given emotion; and it affords the actor considerable control - potentially automatic or deliberate control - over these experiences.

Considering the attenuated reactivity of the psychopathic individual through the lens of this instrumental approach offers a unique perspective on the disorder. For instance, an instrumental lens may cast the psychopathic individual's reduced processing of negativelyvalent/aversive stimuli as indicative of a specific information-processing strategy, intentionally even if ultimately habitually - engaged, which serves to regulate their exposure to aversive stimuli at a level that reflects the utilitarian value placed on that stimulus. If little value is 
expected, the resultant response may become one of indifference; if negative value is expected, the resultant response may become one of active avoidance. In either case, the result may be reduced allocation of processing resources toward the aversive stimulus. We thus propose that psychopathic individuals' insensitivity to negatively-valent/aversive information may occur as a result of the attachment of either neutral or negative value judgements, which subsequently results in indifference or active avoidance, respectively. We further propose that the reduction in stimulus processing as a result of this indifference/avoidance may mimic that of a true physiological insensitivity and may impose similar influences on downstream affective and cognitive processes. Should this be true, one could conceivably conceptualize a dual-process framework, wherein psychopathy could manifest as a result of core emotional/cognitive deficits or through the attachment of reduced value judgements to negative/aversive information (see Fowles and Dindo, 2009, for another presentation of a dual-process model, wherein either low fear temperament and regulatory dyscontrol, may independently predispose to the manifestation of reduced fear).

Recent work delineating the complex interplay between motivation, emotion and attention adds further credence to this proposal (e.g. Engelmann \& Pessoa, 2014; Padmala \& Pessoa, 2014; Raymond \& O’Brien, 2009; Walsh, Carmel, Harpur \& Grimshaw, 2018). Collectively, this work has indicated that changes in motivational salience (e.g. through the presence of reward or punishment) can indeed hold significant influence over the allocation of processing resources within complex environments. In some of this work, motivation has been shown to interact with attentional processes, towards shared control over the processing of presented stimuli. For instance, Engelmann \& Pessoa (2014) have demonstrated that the presence of reward can improve orienting and reorienting towards exogenous cues, at least in part through 
a sharpening of attention; and Walsh, et al. (2018) have demonstrated that increased motivation can promote reduced distractibility from both positive and negative emotional stimuli (see also Padmala and Pessoa, 2014). In other work, the influence of motivation on resource allocation has been shown to be completely independent of attention, such that both motivation and attention invoke separate influence over stimulus reactivity (see Raymond, 2009; Raymond \& O'Brien, 2009). These findings make plausible the motivational framework presented within, and provide testable interactive and independent pathways through which the psychopathic individual's core features could manifest as a result of motivational factors (see Methods for Testing a Motivational Framework below for elaboration).

In contrast to emotion-deficit and cognitive-deficit theories, the motivational framework proposed within should predict that psychopathic individuals would show capacity for typical emotional experiences/reactivity when motivational demands are sufficiently strong. Work in this area is sparse, however, several studies do suggest that the psychopathic individual's sensitivity to aversive stimuli increases when incentive motivation to process those stimuli is increased. Schmauk (1970) demonstrated normalization of anticipatory arousal when psychopaths were threatened with monetary loss (see also Pfabigan et al., 2011); Hare \& Thorvaldson (1970) reported normalized detection of electrical stimulation when bribed with a desired incentive (i.e. cigarettes); and Steuerwald (1996) reported normalized affective facilitation on a lexical decision task following provocation into anger. Note the varied nature of the motivational manipulations, spanning punishment, reward, and arousal - it may be that the balance between approach/avoidance motivations will best predict subsequent behavioral outcomes (see Fowles, 1980 for an elaborated discussion of these ideas, in line with Gray's (1970) original BIS/BAS framework). In addition, the results of at least one study suggest that 
decreases in the psychopathic individuals' responsivity to aversive stimuli may occur as a function of their perceived control over that stimulus (Ogloff \& Wong, 1990). Finally, recent work from our lab (Arbuckle \& Shane, 2017; Shane \& Groat, 2018) and others (Meffert et al., 2013) have demonstrated that psychopathic individuals can show increased emotional experience (compared to their own baseline levels) when motivated through exogenous instruction to do so (see also Drayton et al., 2018). While admittedly limited at present, this research suggests that when sufficiently motivated - via threat, incentive, perceived need, or exogenous instruction the psychopathic individual may be capable of normative responsivity patterns.

Ideas regarding motivational influences on psychopathy are novel, but not without precedent. Perhaps the earliest presentation of similar ideas was put forward by Hare (1978) who suggested that psychopathic individuals may manifest a relatively automatic coping response to "gate out" the impact of anticipated threat. In line with this perspective, Shane \& Peterson (2004) hypothesized that psychopaths may make use of similar defensive strategies to minimize the aversive impact of negatively-valent stimuli. In a somewhat reversed experimental paradigm, these authors demonstrated that defensive-copers (as assessed via a validated self-report methodology) showed learning deficits on Newman's passive-avoidance go/no-go task (cf. Newman, et al., 1989) that paralleled those displayed by psychopathic individuals. That is, those who make use of defensive posturing in the face of aversive/noxious stimuli made more passive avoidance errors, and showed reduced reflection on negative feedback. While admittedly indirect, Shane and Peterson argued that similar nature of the psychopathic individual's and the defensive coper's response styles suggested the possibility of intriguing parallels. In addition, other scholars have put forth hypotheses that overlap considerably with the ideas discussed herein. For instance, Garofalo \& Neumann (2018) have suggested that the motivations 
underlying psychopathic individuals' regulation of their emotions may be distinct, such that they may attach increased value to certain emotional states (e.g. spite and contempt, Garofalo, Neumann, Zeigler-Hill, Meloy, 2019; see also Garofalo, Lopez-Perez, Gummerum, Hanoch \& Tamir, 2019). While these authors did not propose an overarching theory of psychopathy, the idea of differential value attachment to emotional experiences is highly compatible with the ideas discussed here. Additionally, Lynam \& Miller (2015) have suggested that antagonism may exist as an important component of the antisocial personality, and may motivate the psychopathic individual towards cruel, callous behaviors. While seemingly distinct from the defensive/indifference motives we have focused on in this paper, Lynam and Miller's broader suggestion that motivated features may underlie the psychopathic disorder exists as a common theme. Moreover, antagonism may itself stem from the attachment of reduced motivational value to other's emotions; or may itself hold perceived utility for the psychopathic individual. Finally, Vitale et al. (2018; see also Kosson et al., 2018) have put forth two iterations of their 'affect regulation theory' which together provide an increasingly comprehensive theory of the emotionprocessing difficulties in psychopathic individuals. Their theory shows considerable convergence with the motivational framework proposed within, but focuses greater attention on the potential developmental antecedents of the psychopathic individuals' purported avoidance strategies. Of particular importance, they have suggested that these strategies may develop as a response to early stress exposure, and develop over time into a habitual blunting of emotional experiences. We are sympathetic to many of the arguments laid out by Vitale et al. (2018) and Kosson et al. (2018); however, these theories do differ from the proposed motivational framework in at least two ways. First, whereas their affect regulation theory focuses specifically on the potential for active avoidance, the motivational framework provided within intends to serve as a broader 
exploration of the ways in which differential motivation may influence the psychopathic individual's processing strategies. Second, while Vitale et al. (2018) explicitly suggest that the psychopathic individual's coping strategies develop in childhood following explicit stress exposure, the current motivational framework does not presume that abnormal childhood experiences are a prerequisite for this lack of affective responding. Rather, we suggest that this motivation could conceivably occur independent of a specific stressor, and may develop as either innate or learned processing predispositions.

\section{Reconceptualizing Fundamental Characteristics of Psychopathy within a Motivational}

\section{Framework}

One of the strengths of existing theories of psychopathy is their ability to account for a wide variety of the emotional, cognitive and behavioral characteristics of the disorder. Yet as we will show below, a motivational framework for psychopathy may be equally capable of accounting for these same features. To this end, in the next section, we review some of the most well-established empirical findings regarding psychopathy, and suggest how they may be conceptualized within a motivational framework. Our intention is not to provide an exhaustive review of the field, but rather to highlight some of the core features of the disorder, with the strongest empirical backing, to provide examples of how these features may be productively reformulated within a motivational framework.

Attenuated Responses to Aversive Stimuli. The psychopathic individual's attenuated response to aversive stimuli runs deep, and appears characteristic of both subjective and objective reactivity. Whereas existing theories have interpreted these attenuations as indicative of core affective hyporesponsivity (e.g. Lykken, 1957; Fowles, 1980) or core incapacity to optimally allocate attention (e.g. Newman et al., 1990; Hoppenbrouwers, Bulten \& Brazil, 2016), a 
motivated framework may instead posit reduced inclination to fully process aversive stimuli. This reduced inclination could manifest as either indifference (i.e. low approach and avoidance motivations) or active avoidance. While these motivational stances have garnered only minimal experimental attention to date, supportive evidence does exist. For instance, as previously described, several studies have shown that psychopaths' responsivity to aversive stimuli will normalize when incentive motivation to process those stimuli is increased (Hare \& Thorvaldson; Schmauk, 1970; Steuerwald, 1996; Pfabigan et al., 2011), or when explicit instructions request dedicated effort (Meffert et al., 2013; Arbuckle \& Shane, 2017). Perhaps more telling, however, is evidence suggesting that the psychopaths' responsivity to aversive information may be better characterized as aberrant rather than absent (e.g. Hare, 1978; Intrator et al., 1997). A particularly informative example of this was reported in Hare, Frazelle \& Cox (1978), who identified reduced skin conductance, yet increased heart rate, in psychopathic individuals awaiting an impending punishment. In considerable harmony with a motivated framework, Hare (1978) suggested that the increased heart rate may have been indicative of attempts to cope with the incoming threat, and the decreased skin conductance as evidence of the success of the coping attempt. Consistent with this notion, James Gross's highly influential model of emotion regulation has carefully delineated regulatory strategies into antecedent-focused (which occur prior to stimulus awareness) and response-focused (which occur following stimulus awareness) varieties (Gross, 1998a), and has demonstrated that response-focused strategies often engender a counterproductive increase in stress-related physiology (Gross, 1998b). While Hare et al.'s (1978) study predated this regulatory framework, one retrospective interpretation of this study may be that the evoked physiological response was early evidence of the engagement of a response-focused regulatory strategy. Consistent with this notion, in the only known replication/extension of Hare 
et al. (1978) to date, Ogloff and Wong (1990) confirmed the original physiological response pattern - but only when the impending punishment was inescapable (i.e. when no other method of avoidance was available). Emotion-deficit and cognitive-deficit theories of psychopathy should see no need for coping responses of this nature; their mere existence would thus suggest that the psychopaths' attenuated responsivity may be less a matter of core attenuation than commonly conceptualized (see also Vitale et al., 2018; Kosson et al., 2018; Shane \& Peterson, 2004).

Fear-potentiated Startle. Fear-potentiated startle has received particularly dedicated attention in the psychopathy literature, in part because of its ostensible targeting of early, potentially automatic processing (Öhman \& Soares, 1994). While some differential reports exist (e.g. Pastor et al., 2003) the majority of work in this space indicates that psychopaths (particularly those high on Factor 1) show normal eyeblink startle while viewing positive and neutral images, but reduced startle potentiation while viewing negative images (e.g. Vanman, Mejia, Dawson, Schell \& Raine, 2003; Levenston, Patrick, Bradley \& Lang, 2000; Patrick, Bradley \& Lang, 1993). The normal positive/neutral startle is interpreted as evidence of broadly intact autonomic responsivity; the reduced potentiation during negative picture viewing as evidence of a specific deficit in the manifestation of fear-related physiology (see Patrick, 1994).

Of importance, however, psychopaths' startle tends to be reduced compared to neutralpicture viewing, rather than identical (as would be predicted by a true absence of fear-potentiated startle; e.g. Vanman, et al., 2003; Levenston, et al., 2000; Patrick, et al. 1993). Thus, here too the psychopath's response may best be characterized as aberrant, rather than absent. An alternate interpretation may thus be that the high psychopathy group was sensitive to the negative stimuli, but manifested reactions that engendered decreased, rather than increased, startle magnitude. 
Some scholars have suggested this may indicate pleasure in processing the aversive stimuli (Hosker-Field, Gauthier \& Book, 2016; i.e. the "fear enjoyment hypothesis"), since the psychopathic groups' startle tends to be similar during positive and negative picture viewing (e.g. Patrick et al., 2003). Another possibility, consistent with the motivational framework described within, is that the reduced startle during negative picture viewing is indicative of deliberately down-regulated emotion-related physiology that serves to minimize the impact of the aversive stimulus. Such down-regulation could plausibly represent an active coping response (see Hare, 1978), the application of different emotion goals (cf. Tamir et al., 2016; see also Garofalo et al., 2019), and/or the result of a motivation-induced sharpening of selective attentional resources (c.f. Engelmann \& Pessoa, 2014). Consistent with each of these possibilities, Newman, et al. (2010) recently reported normalized fear-potentiated startle when explicit processing of fear-related stimuli was encouraged. Whereas cognitive-deficit theories posit deficiencies in the automatic allocation of attention, we suggest that the encouraged attentional instructions may themselves guide/alter motivational systems important for the prioritization of attentional resources within complex environments (see Todd et al., 2012; 2014, and discussion below, for evidence of motivationally-based prioritization of this nature).

Low Empathy/Guilt. The psychopathic individual's real-world behaviors attest to their low empathy/guilt, which is also consistently demonstrated within laboratory settings (e.g. Domes et al., 2013; Pfabigan et al., 2015; Marsh et al., 2013). While emotion-deficit theories argue for an inability to recognize, appreciate or concern one's self with another's feelings, evidence of normalized empathy in psychopathic individuals following guided instruction (e.g. Arbuckle and Shane, 2017; Meffert et al., 2013) complicates this perspective. Alternately, a motivational framework may propose that the psychopathic individual merely devalues the sharing of others' 
emotions, unless sufficient motivations to do so are in place. Importantly, while emotion-deficit models may conceptualize reduced motivation as stemming from a low ability to recognize the emotional stimulus, a motivational framework would consider motivational deficits as a proximal cause.

At first blush, the notion that the psychopathic individual may simply lack the motivation to share others' emotions may appear overly-simplified; however, we note that interpretations of this nature are commonplace in parallel forensic fields. For instance, the lack of empathy that sexual offenders report for their victims is commonly theorized as evidence of the judicious use of cognitive distortions to prevent full acknowledgement of their victims' perspective (e.g. Marshall, Hamilton, \& Fernandez, 2001). Moreover, contemporary models of empathy have themselves begun highlighting the motivational nature of the construct. Keysers and Gazzola (2014) have suggested that one need consider both the ability and propensity to empathize; Cameron et al. (2015) has explicitly conceptualized empathy as a choice; and Cameron et al. (2017) has demonstrated that even normative populations will freely choose not to empathize with a stranger to conserve the emotional/cognitive resources that doing so would require.

Within this context, we propose that the psychopathic individual may fall low on the spectrum of 'motivation to care', rather than of 'capacity to care' (however, recent unpublished work from our lab suggests that undergraduate students with heightened psychopathic traits may simply show different motivations to care (O’Connell, 2018)).

\section{Attentional Deficits and Normalized Affective Processing Following Proscribed Attention.}

Increasingly compelling work indicates that psychopathic individuals also presents with a set of distinct cognitive abnormalities, including insufficient reflection following punishment (when reward contingencies are also present (Newman, Patterson, Howland \& Nichols, 1990)) and 
decreased distractibility by set-incongruent information (Baskin-Sommers, Curtin \& Newman, 2011). Emotion-deficit theories argue, somewhat awkwardly, that these abnormalities exist as information-processing consequences of the psychopath's reduced sensitivity to aversive and/or emotionally-valent stimuli. However, several recent demonstrations of attentional abnormalities to non-emotional stimuli (e.g. Hiatt, Schmitt \& Newman., 2004), and of normalized emotional processing following proscribed attention (e.g. Dargis, Mattern \& Newman, 2017), have proven increasingly challenging for emotion-deficit theories to incorporate. Thus, more recent explanations have posited specific, largely automatic, attentional deficits that preclude optimal attentional allocation to goal-incongruent information (e.g. Patterson \& Newman, 1993; Hamilton et al., 2015).

A motivational framework of psychopathy may converge with these cognitive-deficit theories in predicting capacity for emotional processing following sufficient attentional allocation. However, whereas cognitive-deficit theories posit subtle cognitive deficits, a motivational framework may more straightforwardly conceptualize the psychopath's attentional biases as indicative of a strategic, if often habitual, attentional selection process. Indeed, as discussed above, substantive work in normative populations demonstrates that attentional allocation can be guided by motivational goals to favour certain stimulus categories over others (c.f. Engelmann \& Pessoa, 2007; Padmala \& Pessoa, 2014, Raymond \& O’Brien, 2009). And seminal work by Todd and colleagues (e.g. Todd et al., 2012; 2014) has a) linked motivationallydriven attention to the instantiation of broader regulatory processes, and b) indicated that these motivational influences can engage and coordinate at the level of early cue processing stages of visual attention. Referred to as 'affect-biased attention', this process is described as an implicit, habitual pre-tuning of sensory systems, such that certain categories of affectively-salient stimuli 
are perceived over others. Evidence for this form of attentional pre-tuning is particularly evident within individuals with diagnosed anxiety/mood disorders, who show robust pre-tuning towards environmental stimuli congruent with their motivational stance (Mogg \& Bradley, 2005; Shane \& Peterson, 2004b). Importantly, whether these anxiety-related biases constitute a cause or consequence of anxiety/mood disorders remains an open, and hotly-debated topic (see Van Bockstaele, et al., 2014 for critical review). Cognitive-deficit theories posit the psychopathic individual's biased attentional allocation as cause: they engender the subsequent devaluation of information that falls outside the current goal-directed set. A motivated framework for psychopathy may instead posit the psychopathic individual's biased attentional allocation as consequence: as a downstream effect of their unique motivational composition. This perspective benefits from consistency with Todd et al.'s (2014) conceptualization of affect-biased attention, which they stress should not be conceptualized as a reactive emotional process, but rather as an early-state, proactive shaping of perceptual experiences in the service of broader regulatory control. One possibility is that through proactive shaping of this nature, motivational differences in psychopathic individuals could appear as attention-based abnormalities.

Passive-Avoidance Learning. Considerable work has documented psychopaths' inferior passive-avoidance learning (e.g. Patterson and Newman, 1993), and important work by Newman and colleagues has convincingly related these deficits to a reduced tendency to stop/reflect following goal-directed error (e.g. Newman et al., 1990). Indeed, whereas non-psychopathic individuals commonly pause following error, psychopaths either fail to pause, or at times speed up. Note, however, that the psychopaths' speed up following error could be interpreted as another example of aberrant - rather than absent - response to an aversive stimulus. Moreover, as reported above, Shane and Peterson (2004) demonstrated that 'defensive copers' manifest 
similar speed/accuracy patterns. This latter finding converges with other work indicating that 'defensive copers' also show reduced fear-potentiated startle (Temple \& Cook, 2007), increased pain tolerance (Jamner \& Schwartz, 1986), and reduced neural response to fearful faces (Rauch et al., 2007). The overlap with core features of psychopathy may (or may not) be circumstantial; at the least, however, they provide another suggestion of how the consequences of reduced processing of aversive stimuli may manifest through multiple pathways. It may indeed be productive to consider the possibility of dual-pathways in the context of psychopathy, wherein either automatic, fear-insensitive disinhibition, or strategic, anxiety-sensitive defensive behavior, could engender the psychopaths' reduced processing of aversive information. The predication of an anxiety-sensitive pathway is in accord with recent theorizing by Vitale et al. (2018; see also Kosson et al., 2018); however, whereas these authors posit an explicit relationship with significant environmental stressors (potentially in childhood), we are more agnostic to innate/environmental antecedents: one could presumably be born high on a spectrum of defensiveness; alternately, one could develop habitual strategies for dealing with aversive stressors.

\section{Testing the Validity of a Motivational Framework}

A strength of this motivational framework is its ability to stimulate novel empiricallytestable hypotheses capable of differentiating from emotion-deficit and cognitive-deficit theories. For instance, whereas deficit-based theories would hypothesize generally consistent reductions in emotion-/attention-related reactivity, a motivational framework may anticipate these reductions to show considerably greater interindividual and contextual mediation. Indeed, if the psychopathic individual's attenuated reactivity is a function of the unique motivational value they place on negative/aversive information, then we may anticipate that a) interindividual 
differences in subjective motivational salience should track with level of reactivity, and b) manipulations aimed at altering the motivational value of a given stimulus should have downstream influence on reactivity levels. To our knowledge, little to no work has yet been undertaken in this space; and we detail below a few avenues that we believe may be fruitful. Parsing Emotion, Attention and Motivation. Perhaps the most direct method for testing assumptions of the motivational framework would be to administer tasks to psychopathic individuals that have proven capable of parsing the influence of emotional, motivational and attentional processes on reactivity to exogenous stimuli. For instance, Engelmann \& Pessoa (2014) successfully parsed motivational/attentional influences through use of a spatially cued forced-choice localization task under varying levels of motivation; Walsh et al. (2018) successfully parsed motivational/emotional influences by evaluating reaction time to positive, negative and neutral stimuli under varying levels of reward incentive; and Raymond and O'Brien (2009) established independence of motivational/attentional influences by measuring recognition of briefly presented faces seen previously in a value-learning task involving monetary wins and losses. To our knowledge, none of these tasks have yet been applied to work on psychopathy, but anticipate the results of such studies being informative.

Consider what specifically motivates the psychopathic individual. The vast majority of work conducted to date has compared reactivity of psychopathic/non-psychopathic individuals to stimuli known to elicit potent reactivity in non-psychopathic individuals. If, however, the psychopathic individual attaches motivational value differently than non-psychopathic individuals, then this previous work may be unintentionally stacking the deck, so to speak. Instead, we may suggest that researchers consider carefully the unique motivational stances of the psychopathic individual, and present stimuli deemed most likely to elicit a reaction in that 
population. As preliminary suggestions: perhaps competitive situations, or situations that afford manipulation of others would motivate their antagonistic tendencies (c.f. Miller \& Lynam, 2005; O’Connell, 2018). Alternately, researchers may consider asking each participant for help in defining what would be motivating for them, and then provide individually-targeted stimuli within the testing environment.

Timing issues. It may also be fruitful to evaluate the specific time-course of participants' reactions to negative/aversive stimuli. This could be accomplished most easily by measuring the time-course of participants' (neuro)physiological responses. Emotion-deficit theories are likely to anticipate stable, low reactivity through the presentation of an aversive stimulus; cognitivedeficit theories may anticipate similarly stable, low reactivity throughout stimulus presentation, but only once a dominant response set has been established; a motivational framework may anticipate a quite different time-course, however: if psychopathic individuals are driven by avoidance motives, we may expect immediately increased reactivity (indicative of underlying sensitivity), followed by subsequently reduced reactivity (indicative of motivated avoidance). Computational Models: Interactions and Dual-pathways. Finally, with larger datasets and emerging computational methods on the horizon, we may be approaching a new opportunity to test finer-grained distinctions regarding the psychopathic disorder than traditional methods, and smaller samples, have previously afforded. As but a few examples, computational methods may be capable of evaluating models that hypothesize interactive effects between motivation, emotion and attention; or that hypothesize the existence of psychopathic subtypes; or of multiplepathways towards the psychopathic disorder (see Brazil et al., 2018 for a recent attempt to use computational modeling to distinguish psychopathy from ASPD; and Fowles \& Dindo, 2005 or Shane \& Peterson, 2004 for early notions regarding dual pathways). As sample sizes and 
computational power continue to grow, these opportunities are likely to become increasingly powerful, and available.

\section{Implications for Treatment and Violence Reduction}

Several early notions posit the psychopathic individual as particularly resistant to treatment (e.g. Cleckley, 1941), and the effectiveness of current treatment modalities may best be described as inconsistent (e.g. Salekin, 2010). While a handful of recent programs have reported early empirical success (e.g. Caldwell, McCormick, Wolfe \& Umstead, 2012; see also Polaschek, 2014 for promising treatment ideas), the ineffectiveness of most programs to date suggest that new treatment paradigms may be beneficial. The majority of programs have focused on targeting purported deficiencies in emotional experience (but see Wong \& Olver, 2015 for a different approach based on risk-need-responsivity principles). However, if the characteristics of psychopathy are a reflection of motivational value judgments, then future treatment modalities may wish to target processes more directly involved in the creation and maintenance of these utilitarian constructs.

We have suggested that psychopathic individuals may attach either neutral or negative value judgements to negatively-valent/aversive information, resulting in indifference, or active avoidance, respectively. The treatment implications in each of these instances may be unique. If psychopathic individuals are primarily characterized by indifference, then there may be an opportunity for unique treatment protocols to focus on increasing the instrumental value attached to negative/aversive stimuli. With this in mind, it is worth noting one recent treatment program that has shown high promise for reducing psychopathic characteristics by focusing on motivating prosocial behavior by providing in-facility incentives to youth with psychopathic traits (Caldwell et al., 2012) and violent behaviours (Caldwell, Skeem, Salekin \& Van Rybroek, 2006). The 
extent to which these methods (i.e. using exogenous incentives) would be equally

feasible/efficacious outside a treatment facility is difficult to know; the holy grail may be to identify techniques capable of increasing motivational value endogenously (though this may be as difficult to identify as the grail itself).

Alternately, if psychopathic individuals are primarily characterized by active avoidance, novel treatment methods may aim at decreasing that avoidance response. Of primary import would be to determine the motivation underlying the avoidance response. Within this manuscript, we primarily highlight defensive motives, but antagonistic tendencies (e.g. contempt) can also relate to avoidant action tendencies. If defensive, insights for treatment opportunities may be garnered from other literatures. For instance, attempts to decrease avoidance tendencies in phobias have made successful use exposure therapy to extinguish conditioned aversive responses (Choy, Fyer, \& Lipsitz, 2007). Likely the translation to psychopathy will not be quite so direct; nonetheless, we know of no research that as ever aimed to even approach the idea of exposure therapy for psychopathy, so opportunities for novel insights in this domain appear potentially rich.

\section{Conclusion}

Clinical and empirical work have on psychopathy have largely converged in assuming broad-scale deficits in either emotional or cognitive processes, that preclude their ability to respond normally to negatively-valent/aversive stimuli. Despite this consensus, there is virtually no research to date to directly support this 'deficit' model. In this paper, we have presented an alternative framework that suggests the psychopathic individuals' core features may stem instead from differences in the motivational value that they attach to aversive information. The 
framework offers well-defined, testable hypotheses, and may offer new ways of conceptualizing the disorder, and of potential next-generation treatment opportunities. 


\section{References}

Arbuckle, N. L., \& Shane, M. S. (2017). Up-regulation of neural indicators of empathic concern in an offender population. Social Neuroscience, 12(4), 386-390. doi.org/10.1080/17470919.2016.1179669

Baskin-Sommers, A. R., Curtin, J. J., \& Newman, J. P. (2011). Specifying the attentional selection that moderates the fearlessness of psychopathic offenders. Psychological Science, 22(2), 226-234.

Baskin-Sommers, A. R., Curtin, J. J., \& Newman, J. P. (2015). Altering the cognitive-affective dysfunctions of psychopathic and externalizing offender subtypes with cognitive remediation. Clinical Psychological Science, 3(1), 45-57.

Blair, R. J. R. (1995). A cognitive developmental approach to morality: Investigating the psychopath. Cognition, 57(1), 1-29.

Blais, J., Solodukhin, E., \& Forth, A. E. (2014). A meta-analysis exploring the relationship between psychopathy and instrumental versus reactive violence. Criminal Justice and Behavior, 41(7), 797-821.

Brazil, I. A., van Dongen, J. D., Maes, J. H., Mars, R. B., \& Baskin-Sommers, A. R. (2018). Classification and treatment of antisocial individuals: From behavior to biocognition. Neuroscience \& Biobehavioral Reviews, 91, 259-277.

Caldwell, M., Skeem, J., Salekin, R., \& Van Rybroek, G. (2006). Treatment response of adolescent offenders with psychopathy features: A 2-year follow-up. Criminal Justice and Behavior, 33(5), 571-596. 
Caldwell, M. F., McCormick, D., Wolfe, J., \& Umstead, D. (2012). Treatment-related changes in psychopathy features and behavior in adolescent offenders. Criminal Justice and Behavior, 39(2), 144-155.

Cameron, D., Inzlicht, M., \& Cunningham, W. A. (2015). Empathy is actually a choice. New York Times, 10.

Cameron, D., Cunningham, W., Saunders, B., \& Inzlicht, M. (2017, March 18). The ends of empathy: Constructing empathy from value-based choice. https://doi.org/10.31234/osf.io/d99bp

Cleckley, H. (1941). The mask of sanity; An attempt to reinterpret the so-called psychopathic personality. St. Louis: The C.V. Mosby Company.

Choy, Y., Fyer, A. J., \& Lipsitz, J. D. (2007). Treatment of specific phobia in adults. Clinical Psychology Review, 27(3), 266-286.

Dargis, M. A., Mattern, A. C., \& Newman, J. P. (2017). Set-congruent priming stimuli normalize the information processing of psychopathic offenders. Journal of Psychopathology and Behavioral Assessment, 39(2), 209-219.

Domes, G., Hollerbach, P., Vohs, K., Mokros, A., \& Habermeyer, E. (2013). Emotional empathy and psychopathy in offenders: An experimental study. Journal of Personality Disorders, 27(1), 67-84.

Drayton, L. A., Santos, L. R., \& Baskin-Sommers, A. (2018). Psychopaths fail to automatically take the perspective of others. Proceedings of the National Academy of Sciences, 115(13), 3302-3307.

Engelmann, J. B. \& Pessoa, L. (2007). Motivation sharpens exogenous spatial attention. Emotion, 7(3), 668-674. 
Fowles, D. C. (1980). The three arousal model: Implications of Gray's two-factor learning theory for heart rate, electrodermal activity, and psychopathy. Psychophysiology, 17(2), 87-104.

Fowles, D. C., \& Dindo, L. (2009). Temperament and psychopathy: A dual-pathway model. Current Directions in Psychological Science, 18(3), 179-183.

Garofalo, C., \& Neumann, C. S. (2018). Psychopathy and emotion regulation: Taking stock and moving forward. In M. DeLisi (Eds.) Routledge International Handbook of Psychopathy and Crime (pp. 76-97). United Kingdom: Routledge.

Garofalo, C., Neumann, C. S., Zeigler-Hill, V., \& Meloy, J. R. (2019). Spiteful and contemptuous: A new look at the emotional experiences related to psychopathy. Personality Disorders: Theory, Research, and Treatment, 10(2), 173-184.

Garofalo, C., López-Pérez, B., Gummerum, M., Hanoch, Y., \& Tamir, M. (2019). Emotion goals: What do sexual offenders want to feel? International Journal of Offender Therapy and Comparative Criminology, $0306624 X 19866114$.

Gray, J. A. (1970). The psychophysiological basis of introversion-extraversion. Behaviour Research and Therapy, 8(3), 249-266.

Gross, J. J. (1998a). The emerging field of emotion regulation: An integrative review. Review of General Psychology, 2(3), 271-299.

Gross, J. J. (1998b). Antecedent-and response-focused emotion regulation: Divergent consequences for experience, expression, and physiology. Journal of Personality and Social Psychology, 74(1), 224-237.

Hamilton, R. K., Hiatt Racer, K., \& Newman, J. P. (2015). Impaired integration in psychopathy: A unified theory of psychopathic dysfunction. Psychological Review, 122(4), 770-791. 
Hare, R. D. (1991). The Hare Psychopathy Checklist-Revised: Manual. Multi-Health Systems, Incorporated. Toronto: ON.

Hare, R. D. (1978). Psychopathy and electrodermal responses to nonsignal stimulation. Biological Psychology, 6(4), 237-246.

Hare, R. D. (1999). Psychopathy as a risk factor for violence. Psychiatric Quarterly, 70(3), 181197.

Hare, R. D., Frazelle, J., \& Cox, D. N. (1978). Psychopathy and physiological responses to threat of an aversive stimulus. Psychophysiology, 15(2), 165-172.

Hare, R. D., \& Thorvaldson, S. A. (1970). Psychopathy and response to electrical stimulation. Journal of Abnormal Psychology, 76(3), 370-374.

Hastings, M. E., Tangney, J. P., \& Stuewig, J. (2008). Psychopathy and identification of facial expressions of emotion. Personality and Individual Differences, 44(7), 1474-1483.

Hemphill, J. F., Hare, R. D., \& Wong, S. (1998). Psychopathy and recidivism: A review. Legal and Criminological Psychology, 3(1), 139-170.

Hiatt, K. D., Schmitt, W. A., \& Newman, J. P. (2004). Stroop tasks reveal abnormal selective attention among psychopathic offenders. Neuropsychology, 18(1), 50-59.

Hoppenbrouwers, S. S., Bulten, B. H., \& Brazil, I. A. (2016). Parsing fear: A reassessment of the evidence for fear deficits in psychopathy. Psychological Bulletin, 142(6), 573-600.

Hosker-Field, A. M., Gauthier, N. Y., \& Book, A. S. (2016). If not fear, then what? A preliminary examination of psychopathic traits and the Fear Enjoyment Hypothesis. Personality and Individual Differences, 90, 278-282. 
Intrator, J., Hare, R., Stritzke, P., Brichtswein, K., Dorfman, D., Harpur, T., et al. (1997). A brain imaging (single photon emission computerized tomography) study of semantic and affective processing in psychopaths. Biological Psychiatry, 42(2), 96-103.

Jamner, L. D., \& Schwartz, G. E. (1986). Self-deception predicts self-report and endurance of pain. Psychosomatic Medicine, 48(3-4), 211-223.

Keysers, C., \& Gazzola, V. (2014). Dissociating the ability and propensity for empathy. Trends in Cognitive Sciences, 18(4), 163-166.

Kosson, D. S., McBride, C. K., Miller, S. A., Riser, N. R., \& Whitman, L. A. (2018). Attentional bias following frustration in youth with psychopathic traits: Emotional deficit versus negative preception. Journal of Experimental Psychopathology, 9(2), 1-21.

Levenston, G. K., Patrick, C. J., Bradley, M. M., \& Lang, P. J. (2000). The psychopath as observer: emotion and attention in picture processing. Journal of Abnormal Psychology, 109(3), 373-385.

Lykken, D. T. (1957). A study of anxiety in the sociopathic personality. The Journal of Abnormal and Social Psychology, 55, 6-10.

Lynam, D. R., \& Miller, J. D. (2015). Psychopathy from a basic trait perspective: The utility of a five-factor model approach. Journal of Personality, 83(6), 611-626.

Marsh, A. A., \& Cardinale, E. M. (2012). When psychopathy impairs moral judgments: neural responses during judgments about causing fear. Social Cognitive and Affective Neuroscience, 9(1), 3-11.

Marsh, A. A., Finger, E. C., Fowler, K. A., Adalio, C. J., Jurkowitz, I. T., Schechter, J. C., ... \& Blair, R. J. R. (2013). Empathic responsiveness in amygdala and anterior cingulate cortex 
in youths with psychopathic traits. Journal of Child Psychology and Psychiatry, 54(8), 900-910.

Marshall, W. L., Hamilton, K., \& Fernandez, Y. (2001). Empathy deficits and cognitive distortions in child molesters. Sexual Abuse, 13(2), 123-130.

Meffert, H., Gazzola, V., den Boer, J. A., Bartels, A. A., \& Keysers, C. (2013). Reduced spontaneous but relatively normal deliberate vicarious representations in psychopathy. Brain, 136(8), 2550-2562.

Miller, J. D., Lynam, D. R., \& Jones, S. (2008). Externalizing behavior through the lens of the five-factor model: A focus on agreeableness and conscientiousness. Journal of Personality Assessment, 90(2), 158-164.

Mogg, K., \& Bradley, B. P. (2005). Attentional bias in generalized anxiety disorder versus depressive disorder. Cognitive Therapy and Research, 29(1), 29-45.

Newman, J. P., Curtin, J. J., Bertsch, J. D., \& Baskin-Sommers, A. R. (2010). Attention moderates the fearlessness of psychopathic offenders. Biological Psychiatry, 67(1), 6670.

Newman, J. P., Patterson, C. M., Howland, E. W., \& Nichols, S. L. (1990). Passive avoidance in psychopaths: The effects of reward. Personality and Individual Differences, 11(11), $1101-1114$.

O’Connell, J. E. (2018). Beyond prosocial motivations to empathize. (Unpublished master's thesis). University of Ontario Institute of Technology, Oshawa, Ontario, Canada.

Ogloff, J. R., \& Wong, S. (1990). Electrodermal and cardiovascular evidence of a coping response in psychopaths. Criminal Justice and Behavior, 17(2), 231-245. 
Öhman, A., \& Soares, J. J. (1994). " Unconscious anxiety": phobic responses to masked stimuli. Journal of Abnormal Psychology, 103(2), 231-240.

Oliver, L. D., Mao, A., \& Mitchell, D. G. (2015). "Blindsight" and subjective awareness of fearful faces: Inversion reverses the deficits in fear perception associated with core psychopathic traits. Cognition and Emotion, 29(7), 1256-1277.

Padmala, S., \& Pessoa, L. (2014). Motivation versus aversive processing during perception. Emotion, 14(3), 450.

Pastor, M. C., Moltó, J., Vila, J., \& Lang, P. J. (2003). Startle reflex modulation, affective ratings and autonomic reactivity in incarcerated Spanish psychopaths. Psychophysiology, 40(6), 934-938.

Patrick, C. J. (1994). Emotion and psychopathy: Startling new insights. Psychophysiology, 31(4), 319-330.

Patrick, C. J., Bradley, M. M., \& Lang, P. J. (1993). Emotion in the criminal psychopath: startle reflex modulation. Journal of Abnormal Psychology, 102(1), 82-92.

Patterson, C. M., \& Newman, J. P. (1993). Reflectivity and learning from aversive events: Toward a psychological mechanism for the syndromes of disinhibition. Psychological Review, 100(4), 716-736.

Pfabigan, D. M., Alexopoulos, J., Bauer, H., Lamm, C., \& Sailer, U. (2011). All about the money-external performance monitoring is affected by monetary, but not by socially conveyed feedback cues in more antisocial individuals. Frontiers in Human Neuroscience, 5(100), 1-12. 
Pfabigan, D. M., Seidel, E. M., Wucherer, A. M., Keckeis, K., Derntl, B., \& Lamm, C. (2015). Affective empathy differs in male violent offenders with high-and low-trait psychopathy. Journal of Personality Disorders, 29(1), 42-61.

Polaschek, D. L. L. (2014). Adult criminals with psychopathy. Current Directions in Psychological Science, 23(4), 296-301. doi:10.1177/0963721414535211.

Rauch, A. V., Ohrmann, P., Bauer, J., Kugel, H., Engelien, A., Arolt, V., ... \& Suslow, T. (2007). Cognitive coping style modulates neural responses to emotional faces in healthy humans: a 3-T FMRI study. Cerebral Cortex, 17(11), 2526-2535.

Raymond, J. (2009). Interactions of attention, emotion and motivation. Progress in brain research, 176, 293-308.

Raymond, J. E., \& O'Brien, J. L. (2009). Selective visual attention and motivation: The consequences of value learning in an attentional blink task. Psychological Science, 20(8), 981-988.

Salekin, R. T., Worley, C., \& Grimes, R. D. (2010). Treatment of psychopathy: A review and brief introduction to the mental model approach for psychopathy. Behavioral Sciences \& The Law, 28(2), 235-266.

Schmauk, F. J. (1970). Punishment, arousal, and avoidance learning in sociopaths. Journal of Abnormal Psychology, 76(3), 325-335.

Serin, R. C. (1991). Psychopathy and violence in criminals. Journal of Interpersonal Violence, 6(4), 423-431.

Shane, M. S., \& Groat, L. L. (2018). Capacity for upregulation of emotional processing in psychopathy: all you have to do is ask. Social Cognitive and Affective Neuroscience, 13(11), 1163-1176. 
Shane, M. S., \& Peterson, J. B. (2004). Defensive copers show a deficit in passive avoidance learning on Newman's Go/No-Go Task: Implications for self-deception and socialization. Journal of Personality, 72(5), 939-966.

Shane, M., \& Peterson, J. (2004b). Self-induced memory distortions and the allocation of processing resources at encoding and retrieval. Cognition and Emotion, 18(4), 533-558.

Steuerwald, B. L. (1996). Anger following provocation in individuals with psychopathic characteristics (Unpublished doctoral dissertation). University of North Carolina at Greensboro, Greensboro, NC

Tamir, M. (2016). Why do people regulate their emotions? A taxonomy of motives in emotion regulation. Personality and Social Psychology Review, 20(3), 199-222.

Tamir, M., Chiu, C. Y., \& Gross, J. J. (2007). Business or pleasure? Utilitarian versus hedonic considerations in emotion regulation. Emotion, 7(3), 546-554.

Tamir, M., Ford, B. Q., \& Gilliam, M. (2013). Evidence for utilitarian motives in emotion regulation. Cognition \& Emotion, 27(3), 483-491.

Temple, R. O., \& Cook, E. W. (2007). Anxiety and defensiveness: Individual differences in affective startle modulation. Motivation and Emotion, 31(2), 115-123.

Todd, R. M., Cunningham, W. A., Anderson, A. K., \& Thompson, E. (2012). Affect-biased attention as emotion regulation. Trends in Cognitive Sciences, 16(7), 365-372.

Todd, R. M., Taylor, M. J., Robertson, A., Cassel, D. B., Doesberg, S. M., Lee, D. H., ... \& Pang, E. W. (2014). Temporal-spatial neural activation patterns linked to perceptual encoding of emotional salience. PloS One, 9(4), 1-11. 
Van Bockstaele, B., Verschuere, B., Tibboel, H., De Houwer, J., Crombez, G., \& Koster, E. H. (2014). A review of current evidence for the causal impact of attentional bias on fear and anxiety. Psychological Bulletin, 140(3), 682-721.

Vanman, E. J., Mejia, V. Y., Dawson, M. E., Schell, A. M., \& Raine, A. (2003). Modification of the startle reflex in a community sample: Do one or two dimensions of psychopathy underlie emotional processing?. Personality and Individual Differences, 35(8), 20072021.

Vitale, J., Kosson, D. S., Resch, Z., \& Newman, J. P. (2018). Speed-accuracy tradeoffs on an affective lexical decision task: implications for the affect regulation theory of psychopathy. Journal of Psychopathology and Behavioral Assessment, 1-7.

Walsh, A. T., Carmel, D., Harper, D., \& Grimshaw, G. M. (2018). Motivation enhances control of positive and negative emotional distractions. Psychonomic bulletin \& review, 25(4), $1556-1562$

Williamson, S., Hare, R. D., \& Wong, S. (1987). Violence: Criminal psychopaths and their victims. Canadian Journal of Behavioural Science/Revue Canadienne des Sciences du Comportement, 19(4), 454-462.

Wong, S. C., \& Olver, M. E. (2015). Risk reduction treatment of psychopathy and applications to mentally disordered offenders. CNS Spectrums, 20(3), 303-310.

Zeier, J. D., Maxwell, J. S., \& Newman, J. P. (2009). Attention moderates the processing of inhibitory information in primary psychopathy. Journal of Abnormal Psychology, 118(3), 554-563. 